\title{
Mathematical Modelling in Analytical Chromatography: Problems and Solutions
}

\author{
A. M. Dolgonosov ${ }^{a}$ *, A. G. Prudkovskii ${ }^{a}$, E. A. Zaitseva ${ }^{a}$, N. K. Kolotilina ${ }^{a}$, and A. A. Dolgonosov ${ }^{a}$ \\ ${ }^{a}$ Vernadsky Institute of Geochemistry and Analytical Chemistry, Russian Academy of Sciences, Moscow, 119991 Russia \\ *e-mail:amdolgo@mail.ru
}

Received April 4, 2021; revised April 15, 2021; accepted April 15, 2021

\begin{abstract}
Based on an analysis of the results of original research performed in the Laboratory of Sorption Methods of the Vernadsky Institute of Geochemistry and Analytical Chemistry of the Russian Academy of Sciences within the project "Mathematical Chromatograph," the review covers the aim and strategy of the imitation modeling of high-performance chromatography; associated problems of the theory of intermolecular interactions; classifications of polar stationary phases by their selectivity; descriptions of the kinetics and dynamics of sorption processes, choice of the composition of multicomponent mobile phases in HPLC and ion chromatography using the method of the dynamic map of a chromatographic system; and the development of alternating gradient modes using a mathematical experiment.
\end{abstract}

Keywords: analytical chromatography, imitation modeling, theory of intermolecular interactions, selectivity of separation, sorption dynamics

DOI: $10.1134 / \mathrm{S} 1061934821110046$

Chromatographic processes are characterized by high requirements to the composition of mobile and stationary phases and to the precision of implementation of complex modes of the separation of multicomponent mixtures. However, in practice, the choice of phases and conditions is often accidental with the parameters far from the optimum ones.

The state-of-the-art theory of sorption dynamics in combination with the results of the physicochemical description of selectivity and kinetics of sorption allow a researcher to make this choice conspicuous and more perfect. The software products developed on the basis of the mathematical modeling of chromatographic processes that take into account their specific features that allow a researcher to perform full-scale mathematical experiments are especially convenient for the search for optimal conditions and new separation modes. Such imitation modeling must implement high-level mathematical models using no adjustable parameters and, therefore, capable of a priori calculations.

For this purpose, we put forward a new concept of chromatographic analysis and developed a corresponding methodology, based on the maximum replacement of real experiments by simulations, performed using high-level mathematical models. Mathematical experiments require solution of the so-called direct modeling problem, which ensures the calculation of the result of a process based on specified input data. With an adequate model, the results of a priori calculations agree well with the results of test experiments. By varying the parameters of an instrument and solving the direct problem for each version, one can choose such values of parameters that satisfy restrictions of the method and the optimality criterion. This is the aim of using a mathematical model for the analysis optimization. The inverse problem of modeling, being an instrument for an analysis of the results of a real experiment, can be posed only at a successful solution of the direct problem. When performing chemical analyses, one should interpret an analytical signal as chemical information. This is a mathematically incorrect procedure, associated with the multiplication of facts based on the assumptions and intuition of an operator and associated with the risk of a false result. The use of the inverse problem of modeling, based on the theoretical knowledge of the features of the applied method, significantly increases the level of accuracy and reliability of the results of analysis. Thus, imitation modeling of chromatography allows a researcher to considerably reduce the volume of experimental work in developing analytical procedures, avoid mistakes in the identification of analytes, and reduce the systematic component of the experimental error.

The general aim of imitation modeling in high-performance chromatography can be subdivided into a number of problems for the development of mathematical models of methods of gas and ion chromatography and HPLC and the development of correspond- 


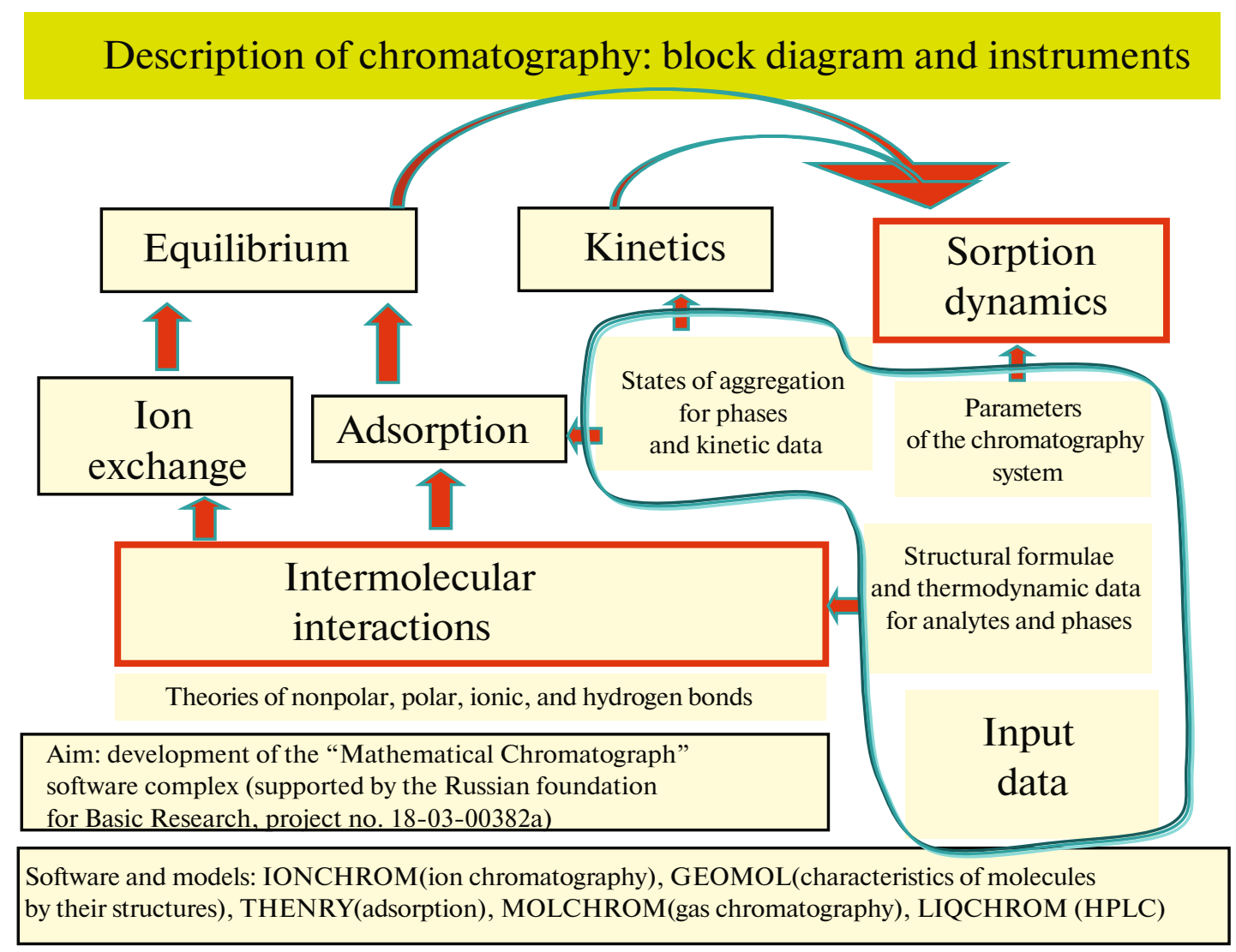

Fig. 1. Block diagram of interrelations in the software for imitation modeling of methods of analytical chromatography in the software complex "Mathematical Chromatograph.”

ing software products. This goal was considered within the initiative project no. 18-03-00382, supported by the Russian Foundation for Basic Research in 20182020 under the conditional name "Mathematical Chromatograph."

Figure 1 presents a flowchart of correlations in the software for the imitation modeling of methods of analytical chromatography taking into account the following features:

- the dynamic process in chromatography follows the laws of quasi-equilibrium thermodynamics and includes equilibrium and kinetic parameters;

- equilibrium is determined by the nature of intermolecular interactions, in accordance with the participation of nonpolar, polar, and ionic interactions and hydrogen bonds;

- kinetics is determined by the values of diffusion constants in the mobile phase (MP) and stationary phase (SP) and by the sizes of the substrate and the SP working area;

- the input data of a model are parameters of the experimental system and the fundamental properties of substances forming the phases and analytes.

\section{MATHEMATICAL MODELS OF ANALYTICAL CHROMATOGRAPHY}

Usually modeling in chromatography is based on abundant empirical data, obtained by the manufacturers of equipment using interpolation methods [1, 2]. Such approaches are laborious, specific to the equipment, and are quite limited in the optimization and search for new modes. There are also semiempirical models, more justified, and to a certain extent capable of predicting the results [3-5]. However, because of a large number of empirical parameters and the inaccuracy of correlations, the search for new, more effective modes with their help is almost excluded. In contrast to them, within the project "Mathematical Chromatograph," we developed and constantly improve high-level models based on the fundamental theoretical description of chromatographic methods of analysis $[6,7]$. Such models open wide possibilities for performing mathematical experiments, also under very complex conditions that cannot be found in the random experimental search.

General dynamic properties of chromatographic processes. The description of a chromatographic process can be conditionally subdivided into two almost nonoverlapping fields: the thermodynamics and the 
macrokinetics of sorption. The relevance of the description of the macrokinetics of chromatography is in many respects associated with the successful solution of the problem of the a priori calculation of a sorption equilibrium. Is it reasonable to calculate peak shape, height, and width if we cannot predict its position in the chromatogram, which is determined by the equilibrium? The consideration of a dynamic problem of high-performance column chromatography, as a first approximation, gives a chromatogram equation in the form of an envelope of Gaussian curves $J(t)$, constructed for the sample components (analytes):

$$
J(t)=\sum_{i} J_{i}(t) ; \quad J_{i}(t)=\frac{\eta_{i} m_{i}}{w_{i} \sigma_{i} \sqrt{2 \pi}} \exp \left[-\frac{\left(t_{i}-t\right)^{2}}{2 \sigma_{i}^{2}}\right],
$$

where $J_{i}(t)$ is the level of signal of the $i$-th component at the instant $t$ in the output section of the column; $m_{i}$ is mass of the $i$-th component in the injected sample; $\eta_{i}$ is coefficient of detector sensitivity to the $i$ th component; $w_{i}$ MP flow rate at the moment of elution of a component peak; and $\sigma_{i}^{2}$ is the dispersion of the peak due to the kinetic properties of the MP, sample component, and structural parameters of the SP.

The position of the maximum of a chromatographic peak in the general case of variable conditions

and a compressible MP is determined as $t_{i}=t_{0}+t_{i}^{\prime}(L)$ from the integral equation:

$$
t_{i}^{\prime}(L)=\int_{0}^{L} \frac{k_{i}\left(t_{i}^{\prime}(x)\right)}{\left(x, t_{i}^{\prime}(x)\right)} d x
$$

where $t_{i}^{\prime}(x)$ is the function obtained as a result of solution of Eq. (2); $t_{0}=\int_{0}^{L} \frac{d x}{V\left(x, t_{i}^{\prime}(x)\right)}$ is dead time; $L$ is column length; $v\left(x, t_{i}^{\prime}(x)\right)$ is linear velocity of the mobile phase in point $x$ at the instant when the component front intersects it; function $k_{i}(t)$ is determined by the boundary conditions from the eluotropic force of the MP and the temperature of the system. In particular, in the isocratic/isothermal mode, $k=$ const, and retention time takes the usual form: $t_{i}=t_{0}\left(1+k_{i}\right)$.

The kinetic characteristics of systems of high-performance chromatography were theoretically well studied and are displayed in terms of the height equivalent to a theoretical plate (HETP) [8], which is described by the van Deemter equation [9]:

$$
h=\frac{A}{V}+B V^{0.5}+C V
$$

(corrected form). Expressions for the coefficients in Eq.(3) are summarized in Table 1. One can see that
Table 1. Coefficients for the contributions of kinetic stages to the height equivalent to a theoretical plate

\begin{tabular}{l|c}
\hline \multicolumn{1}{c|}{ Factor } & Expression for the coefficient \\
\hline Longitudinal dispersion & $A=2 D_{1}$ \\
External diffusion & $B=\frac{\mathrm{const} \cdot d^{1.5}}{\left(1+1 / k_{i}\right)^{2}}$ \\
Internal diffusion & $C=\frac{2 k_{i} r^{2}}{15 D_{2}\left(1+k_{i}\right)^{2}}$ \\
\hline
\end{tabular}

HETP depends not only on hydrodynamic ( $d$ is sorbent mesh, $v$ is linear velocity of the MP) and kinetic parameters $\left(D_{1}, D_{2}\right.$ are analyte diffusion constants in the MP and SP respectively, $r$ is working area of the $\mathrm{SP})$, but also on equilibrium characteristics $\left(k_{i}\right.$ is retention factor related to the Henry constant of the analyte by the phase ratio). Calculations of HETP for each model possess their specific features, the account of which allows a researcher to avoid experiments on the determination of diffusion constants and other empirical parameters [7].

The value inverse to HETP has meaning of the specific efficiency of a column, measured in the number of theoretical plates per unit column length; therefore, the efficiency of the column (number of theoretical plates $N$ ) is determined by the integral

$$
N=\int_{0}^{L} \frac{d x}{h(x)}
$$

Modeling of gas chromatography. Version of gas adsorption chromatography. The instrument proposed in the works $[10,11]$ and most completely described in the monographs $[6,7]$ presents the new nonlinear molecular and statistical (NMS) theory of adsorption in the Henry region, based on the theory of generalized charges (TGC), which was developed based on the general principles of quantum statistics [12-14]. Unlike the semiempirical methods based on the rough postulate of the additivity atom-atomic potentials [15-17], in TGC another summation rule was derived [18-20], because of which an acceptable accuracy of the description of interatomic and intermolecular interactions without using adjustable parameters was attained. The expression for the potential of van der Waals interactions, coinciding with the LennardJones potential in shape and requiring no empirical parameters, was derived from TGC. On this basis, a model of the critical state of substance, extremely important for the theory of first-order phase transitions, was proposed in [21, 22]. Using TGC and the model of the critical state, a relationship between the parameters of intermolecular interactions and critical temperature was considered [23]. On an example of inert and simple gases, ab initio calculations were per- 
formed, the results of which well agreed with the experimental data.

The application of the NMS theory to a priori calculations of the adsorption of several hundred of organic molecules (linear, branched, and cyclic hydrocarbons; oxygen- and halogen-containing compounds) on graphite gave a good agreement with the experimental data on the temperature dependence of the Henry constant for different adsorbates and adsorbents $[6,7,11,14,24,25]$. The precision with which this dependence is known strongly affects a possibility of the prediction of the components behavior under complex conditions of programmable temperature.

Block schemes of the GEOMOL and THENRY software, developed for calculations of the temperature dependence of the partition coefficient by the structural formula of a sample component of test are presented in Fig. 2. However, the resolution of component peaks in a mixture depends not only on their position, but also on their width. The correlations of a dynamic process of chromatography in the model of gas chromatography were complemented with estimates of diffusion coefficients and of HETP as a whole in the description of kinetics [25, 26]. Therefore, the mathematical basis in modeling of molecular chromatography is provided by fundamental theoretical ideas of the adsorption and kinetic mechanisms, implemented in the chromatographic process. Based on the described mathematical fundamentals, the MOLCHROM software, ensuring the solution of the direct problem of modeling of gas adsorption and gasliquid chromatography with nonpolar phases (Fig. 3), was developed.

Version of gas-liquid chromatography. In the description of processes of gas-liquid chromatography, the poorly studied problems are mainly those associated with the imperfection of the characterization of selectivity of polar stationary phases. The characterization of the selectivity of an SP in gas chromatography faces the problem of the determination of desorption energy in the system with dispersion, electrostatic, and hydrogen bonds. For the very important value, the contribution of hydrogen bonds (H-bonds), there still has been no consensus about its nature, and, therefore, value. Based on the conclusions of the traditional quantum theory of chemical bonding about the lack of vacant orbitals for the formation of $\mathrm{H}$ bonds, it was considered after Poling [27] that this bond is of electrostatic rather than quantum nature. Today this version is implemented in models forming the basis of semiempirical methods of calculations of intermolecular interactions (IMI). Contrary to the observed feature of the "classical" $\mathrm{H}$-bond, the longwavelength shift of the IR spectrum of vibrations of the covalent bonds of hydride on the formation of a molecular complex [28], the assumption about the electrostatic nature of the hydrogen bond can only explain the effect of the "blue" shift of the specified spectral band, for which the special term "non-classical" or "improper" hydrogen bond was introduced [29]. The contradiction between the specific nature of the hydrogen bond and electrostatic forces for a particular system is not pronounced, because the reason for these types of interactions is common: the presence of a hydrogen atom and electronegative atoms; the strengths of interactions are also of equal level. Nevertheless, works on hydrogen bonds based on the statistical analysis of empirical data are known, in which electrostatic and covalent forces are independent components [30]. Because of basic falseness of the model considering only dispersion and electrostatic IMI, the convenience of the approaches implemented in computer programs is reduced by the random choice of the parameters of calculations, relating to the set of conformations, fixed angles, and the number of the formed $\mathrm{H}$-bonds, which results in the unreliability of predictions, which, as a result, must be checked and corrected [31, 32].

A fundamental difference between a classical electrostatic interaction of charges localized on atoms and a quantum-mechanical (electron) bond consists in the direct participation of electrons in the formation of the quantum-mechanical bond. The steady state of an atomic system is provided by a finite movement of a hydrogen electron in the region of such a bond; therefore, its kinetic energy cannot exceed the absolute value of the potential energy of the bond. Having reverted this relation, we will obtain that binding energy cannot be lower a certain value dictated by the quantum uncertainty. Therefore, the quantum nature of an H-bond manifests itself in a special effect: the entrance of a molecule in the region of a small solid angle with the vertex on a hydrogen atom of another molecule leads to a jump of the energy of IMI, which can be presented as a drop of a system in a narrow potential well. Therefore, in addition to the effects of polar nature, depending on dipole moments, hydrogen bonding, as dispersion forces, makes an independent contribution to IMI.

The potentials of the approach developed on the basis of TGC were shown not only in a priori calculations of adsorption and van der Waals interactions [18-20], but also in the description of covalent bonds [33-35]. Hydrides are obligatory participants of hydrogen bonds. The accuracy of the developed approach to the description of hydride characteristics was demonstrated in [35]. The derived simple expression for the length of bond in hydrides with a good accuracy corresponds to experimental values for a wide series of chemical elements:

$$
r_{\mathrm{HX}}=3.771 a_{0} \arcsin \sqrt{\frac{2(n-1)^{2}-\sqrt{2}(n-2)}{Z+1}},
$$

where $n, Z$ are the number of the period and atomic number of acceptor $\mathrm{X}$ and $a_{0}$ is Bohr's radius. 


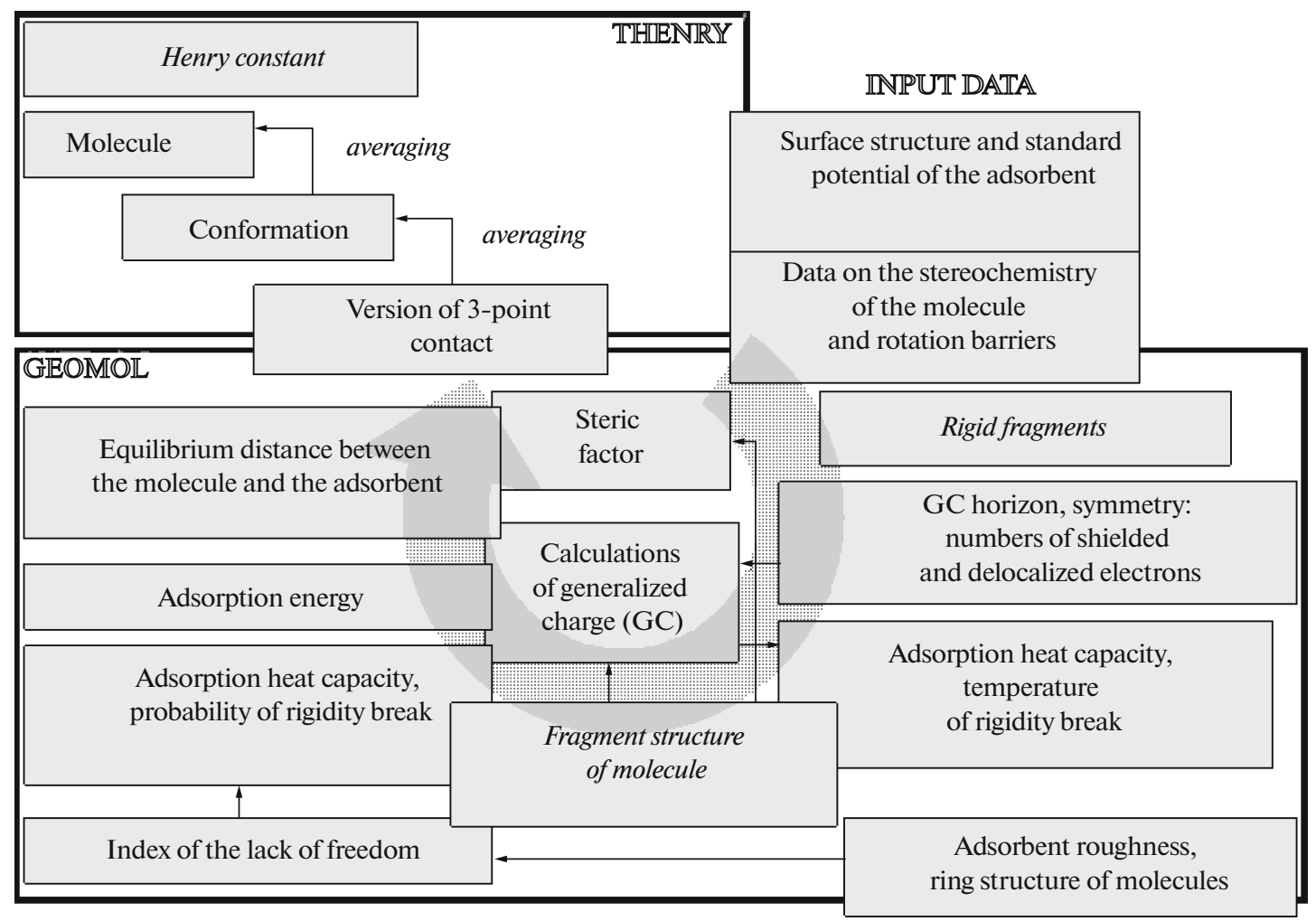

Fig. 2. Operation algorithm of software for modeling adsorption. Arrows show the directions of calculations; superimposing rectangles indicate interrelations between corresponding points.

According to the developed theory of $\mathrm{H}$-bond, the hydride and hydrogen bonds are of common nature, and their characteristics for identical elements are correlated. The regularities of the $\mathrm{H}$-bond were studied in [36], where expressions for its characteristics were derived and criteria of its existence were formulated. The theoretical graph (Fig. 4), constructed on the coordinates $x=\frac{r_{\mathrm{H} \ldots \mathrm{X}}}{a_{0} Z^{1 / 3}} ; y=\frac{2 E_{\mathrm{H} \ldots \mathrm{X}} r_{\mathrm{H} \ldots \mathrm{X}}^{2}}{a_{0} e^{2}}\left(r_{\mathrm{H} \ldots \mathrm{X}}, E_{\mathrm{H} \ldots \mathrm{X}}\right.$ are the length and energy of an $\mathrm{H}$-bond, respectively; $Z$ is atomic number of the acceptor in the $\mathrm{H}$-bond; and $e$ is elementary charge), demonstrates the region of the existence of a hydrogen bond, the borders of which separate classes of substances by their ability to form hydrogen bonds. Based on quantitative estimates, explanations of the presence or absence of solubility in water are given for substances bearing electronegative atoms. It was shown that the energy of a hydrogen bond cannot be lower a certain value:

$$
y \equiv \frac{2 E_{\mathrm{H} \ldots \mathrm{X}} r_{\mathrm{H} \ldots \mathrm{X}}^{2}}{a_{0} e^{2}} \geq \frac{1}{6} \rightarrow E_{\mathrm{H} \ldots \mathrm{X}} \geq \frac{a_{0} e^{2}}{12 r_{\mathrm{H} \ldots \mathrm{X}}^{2}} .
$$

Expressions for the limits of the minimum energy and maximum length of a hydrogen bond through the atomic number of an acceptor can be derived from the theory: $\quad E_{c, \mathrm{H} \ldots \mathrm{X}}=48.94 Z^{-2 / 3} \quad(\mathrm{~kJ} / \mathrm{mol})$, $r_{c, \mathrm{H} \ldots \mathrm{X}}=0.1119 Z^{1 / 3}(\mathrm{~nm})$. The developed theory gives quantitative explanations for some "irregularities," for example, to the effect of the hydrophobicity of chloroalkanes, despite the ability of the hydrogen chloride to hydrogen bonding [37].

In $[37,38]$, the new approach to the description of $\mathrm{H}$-bond, as in the general model of IMI, was expanded to organic matter and to the interaction of vapor molecules with a liquid. The energy of IMI, according to the proposed model, contains three independent groups of values, describing nonpolar and polar forces and hydrogen bonds. Each of forces was presented by its particular molecular descriptor, generalized charge, dipole moment, and two numbers reflecting the ability of a molecule to be a donor or an acceptor of an $\mathrm{H}$-bond, respectively. Using TGC, "structure-property" correlations were obtained for all components of molecular interaction energy. In particular, the contribution of an $\mathrm{H}$-bond to the total energy of IMI as a product of the threshold value (4), being of quantum-mechanical nature, and probabilities of the regular arrangement of interacting molecules $n_{\mathrm{H}}$ were described. The last value was determined as the probability of the entrance of a system of interacting molecules in a narrow potential well and was expressed through the parameters of the molecular structure (Fig. 5). 


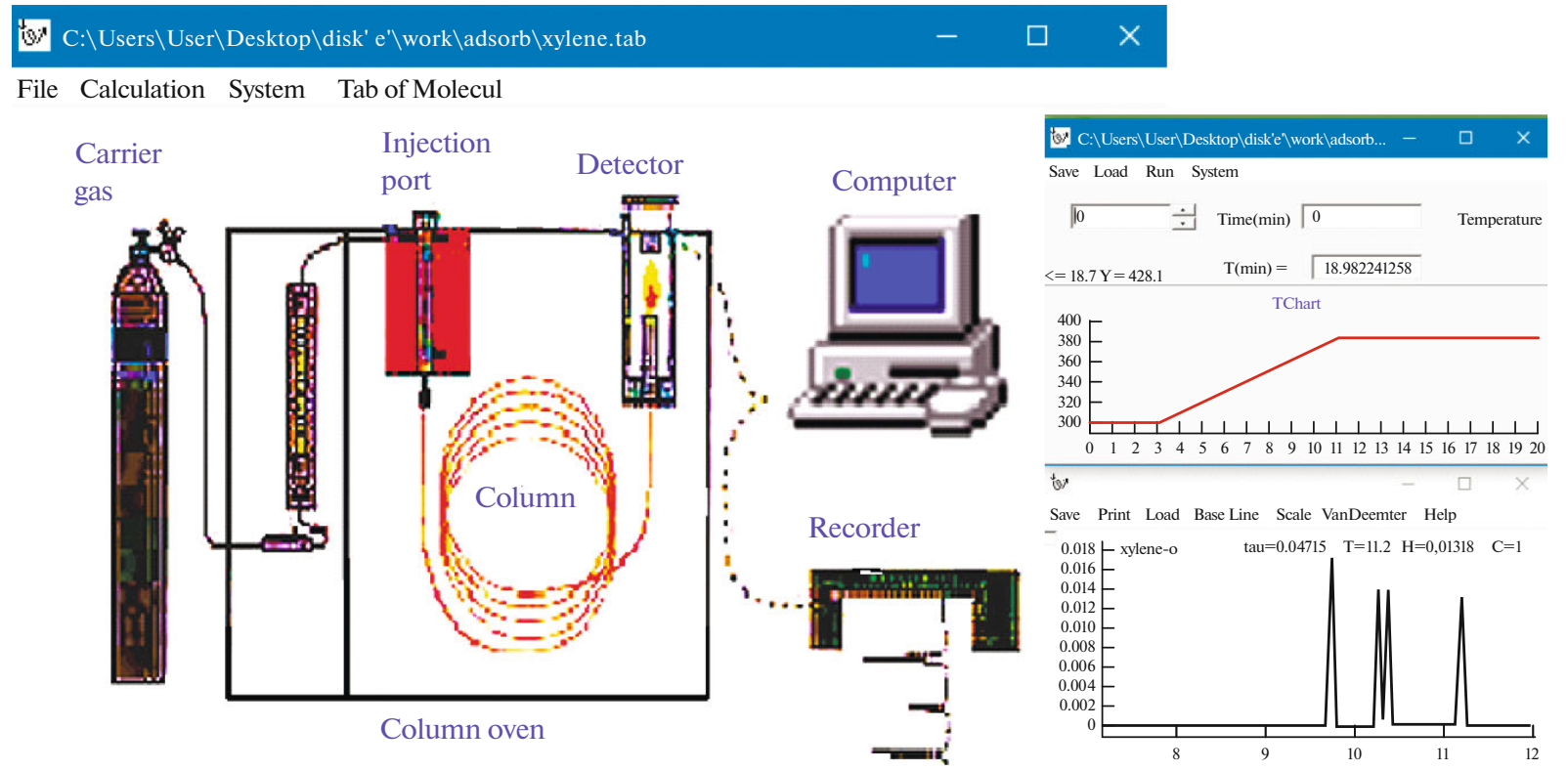

Fig. 3. Interactive interface of the MOLCHROM software with open windows "Oven" and "Recorder". An example of separation (in the order of elution) of styrene; $m^{-} o^{-}$, and $p$-xylenes on a capillary column with a nonpolar phase of the squalene type.

Therefore, new ideas about the H-bond, together with the conclusions of TGC, allowed us to express the energy of IMI of a general type, and, in particular, the energy of adsorption of polar analytes on polar phases, through structural descriptors.

Traditional empirical methods of the classification of stationary phases are known in chromatography $[3,39]$. The five-dimensional Rorschneider scheme (and similar procedures) is based on the anticipated independence of five types of energy for five reference samples. However, "pure" substances, which participate in only one type of interaction, do not exist. In addition, independent types of intermolecular energy are not numerous, i.e., less than five. From here follows the inaccuracy and redundancy of the scheme, because the space of Rorschneider parameters is of lower dimensionality, and the characteristics of reference substances summarize contributions of different types of energy and, therefore, cannot serve as basis vectors of the coordinate system. Other methods [40] utilize the one-dimensional scheme of a hydrophobic-hydrophilic equilibrium, in which stationary phases possess one estimation parameter, which is obviously insufficient because of the complex nature of intermolecular interactions.

The new method of three-parameter characterization (TPC), developed on the basis of the proposed model, is theoretically justified and in balance by the number of independent variables in the description of IMI energies [41]. In the TPC method, two characteristics of an SP, polarity $v=\frac{\mu^{2}}{Q}(Q$ is generalized charge, $\mu^{2}$ is squared dipole moment) and hydrophily
$w=\frac{n_{\mathrm{H}}}{Q}\left(n_{\mathrm{H}}\right.$ is probability of $\mathrm{H}$-bond formation $)$ are determined from three molecular properties (generalized charge, dipole moment, and the parameter of $\mathrm{H}$ bonding), which can be conveniently plotted on a map, which is named the map of phase selectivity. The TPC method allows an analyst not only to perform a priori calculations of the characteristics of phase selectivity, but also to reveal new dependences of these characteristics on the specific features of the molecular structure of phases. In particular, a dependence of the characteristics of polarity and hydrophily of polyethyleneglycol (PEG) on the weight of the polymer molecule was found in [42]. The derived theoretical dependences of the polarity and hydrophily of PEG on molecular weight are presented in Fig. 6 as a parametrical dependence of hydrophily on polarity and well correlate with the experimental data plotted on the selectivity map.

The IMI model was also used in solving another important problem of analytical chromatography, the determination of the conditions and SP parameters of the fine chromatographic separation of the geometrical isomers of fatty acid methyl esters [43]. The inversion of the selectivity of different phases, observed in practice for trans- and cis-isomers, was explained by two sorption mechanisms, one of which was responsible for physical adsorption (and the cis/trans elution order), and the other, for the absorption of the adsorbate by the macromolecule of the polymer phase (and reverse retention order). The last mechanism is more efficient, but requires the obligatory formation of $\mathrm{H}$ - 


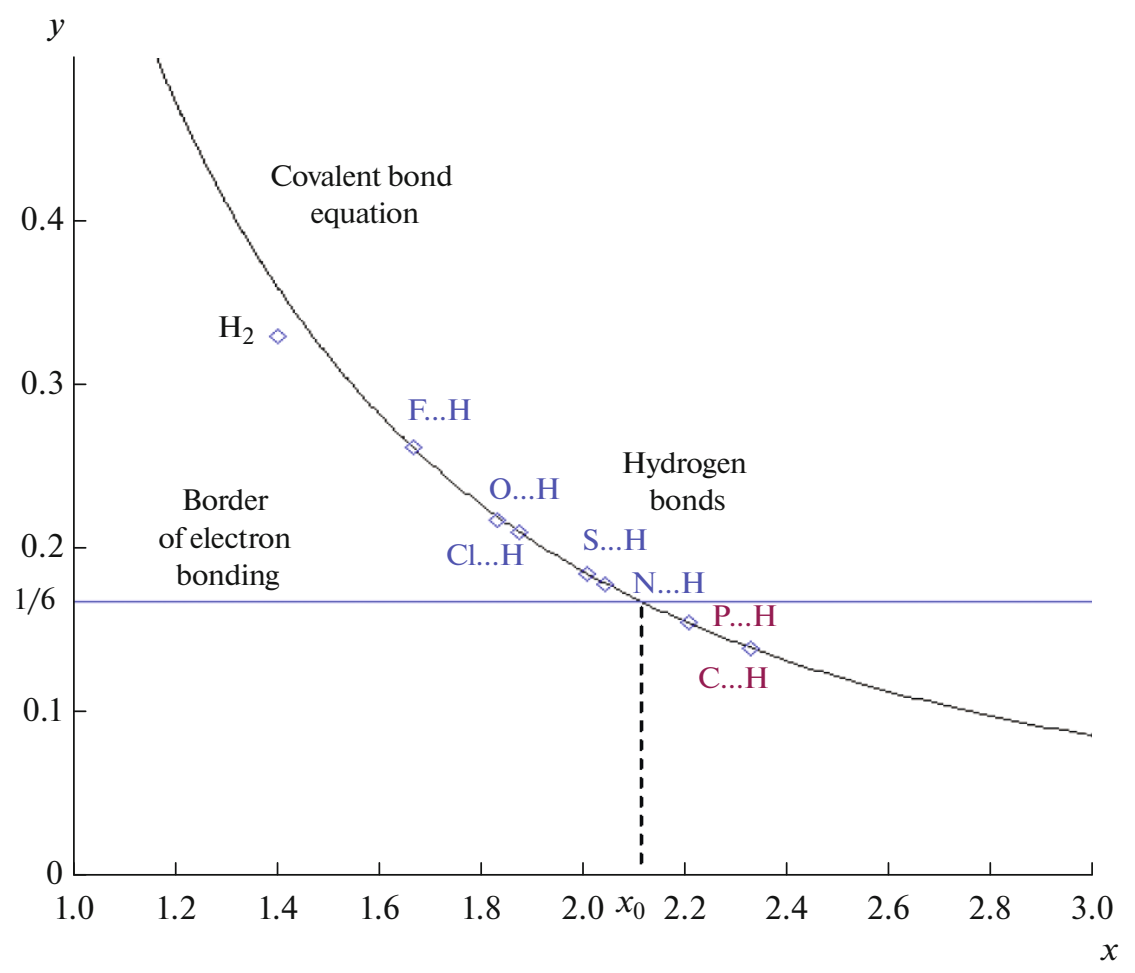

Fig. 4. Region of hydrogen bonds in the "length-energy" plot of electron bonding according to the theory of generalized charges. The point with the coordinates $\left(x_{0}, 1 / 6\right)$ corresponds to dimensionless values of $\mathrm{H}$-bond minimum energy and maximum length.

bonds and a correspondence of the sizes of the macromolecule globule to the adsorbate length.

The attained accuracy of the IMI model opens possibilities for the consideration of the direct modeling problem in gas chromatography: the determination of retention parameters by the specified structures of analytes and the stationary phase. Therefore, the model of gas-adsorption chromatography, developed for nonpolar substances and phases, is supplemented by the description of polar interactions, which ensures the development of a general model of gas-liquid chromatography. The adequacy of the model was checked at different stages of its development. The agreement between the results of calculations for the direct problem and experimental data for gas chromatography is so satisfactory that there appears a possibility for the creation of a simulator program of a stateof-the-art gas chromatograph with wide possibilities in the selection of a carrier gas, a column, a sorbent, test sample components, temperature, and gasdynamic modes.

Modeling of liquid chromatography. In HPLC, problems of the description of competitive sorption, multicomponent eluents, solvation, dissociation, etc., peculiar to ion chromatography, are added to the problems of modeling of gas chromatography. The phenomenological model of HPLC [6, 44], summarizing present-day retention theories [3-5], in combination with TGC allowed us to describe real systems almost without empirical parameters rather well, except for such transferable parameters as sorption capacity and standard energy of the adsorbent. Figure 7 presents an example of a comparison of the results of a priori calculations of the retention factor of naphthalene in the system of reversed-phase HPLC depending on the percentage of modifier in the MP with the experimental data [45].

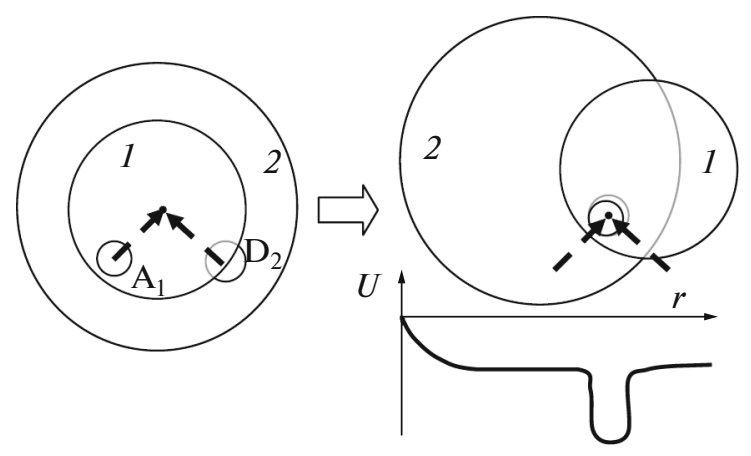

Fig. 5. Molecular cross-sections (circles 1 and 2) with $\mathrm{H}$-bond atoms $\mathrm{A}_{1}$ (acceptor) and $\mathrm{D}_{2}$ (donor) and independent motion of molecules relative to the center of a dispersion interaction (shown by arrows) that must form for the system of two molecules fall in a potential well. The concept of a narrow potential well illustrates the graph for potential energy (shown in the bottom-right corner). 


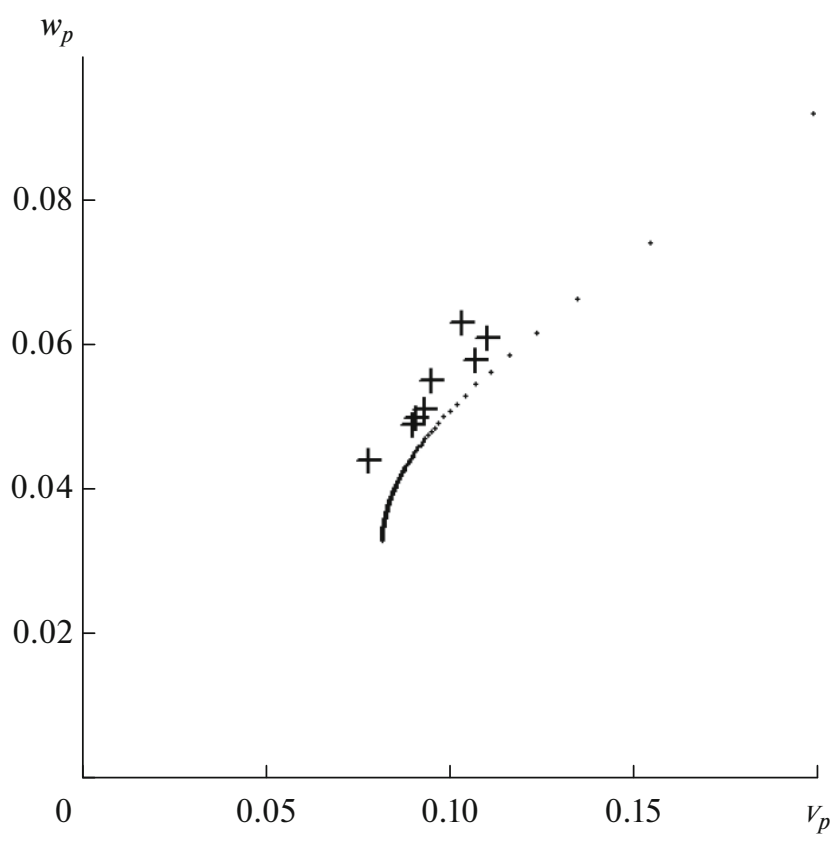

Fig. 6. Plot (selectivity map) on the coordinates polarity $\left(v_{p}, \mathrm{D}^{2}\right)$-hydrophily $\left(w_{p}\right)$ for polyethyleneglycol (PEG) with different weights. Designations: $(+)$ data calculated by the experimental McReynolds constants; dotted curve, theoretically calculated characteristics of PEG; the discrete character of the curve is due to the step corresponding to the monomer weight of $44 \mathrm{Da}$. The shift of the experimental datas relative to the theoretical curve along the abscissa $\left(0.020-0.025 \mathrm{D}^{2}\right)$ is due to the systematic error of the McReynolds method.

The specified model was complemented with an equation for the energy of IMI, derived for polar systems in gas chromatography, for the description of various versions of solvation, affecting retention in HPLC [46]. Because of its extreme complexity, this problem requires further investigation.

An equation for the eluotropic force of a multicomponent MP in liquid chromatography for the case of effective eluents was derived in [47]; it is similar to the equation for an eluent in ion chromatography [48, 49]. Eluotropic force $\varphi$ is a unified characteristic of a multicomponent mixture, similar to the concentration of a single-component MP. It obeys the following equation, common for ion and liquid chromatography:

$$
a_{0}=\sum_{j} n_{j} c_{j} K_{j}^{n_{j}} \varphi^{-n_{j}}
$$

where $c_{j}$ is concentration (taking into account activity coefficient), $K_{j}$ is constant of ion exchange of the $j$ th MP component for the benchmark component "R" (BC) (for HPLC, $K_{j} \equiv K_{j R}$ is constant of competitive sorption of a test component and the $\mathrm{BC}), n_{j}$ is component charge (for HPLC, ratio of molecular areas of a test component and the $\mathrm{BC}), a_{0}$ is the capacity of the SP to BC.

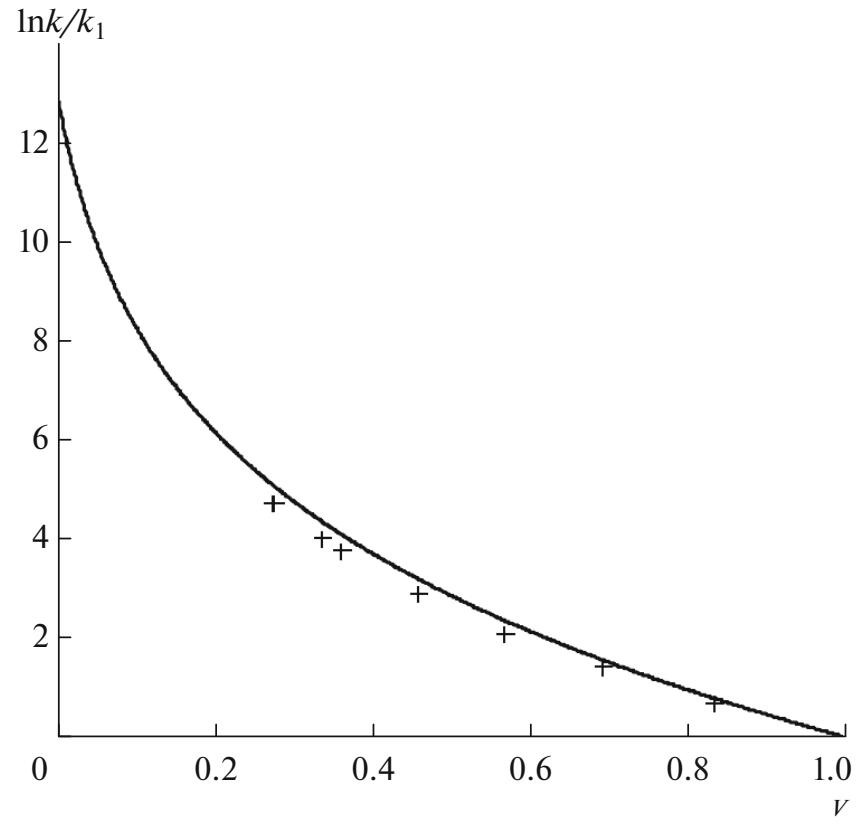

Fig. 7. Logarithm of the retention factor of naphthalene as a function of the percentage of the modifier in the water-methanol mobile phase. Experimental data are shown by points $(+)[45]$ and calculations, by the solid line [44].

Modeling of ion chromatography. The principles and possibilities of an approach to modeling of ion chromatography (IC), based on the achievements of the theory of the dynamics of sorption, adapted to the specific features of ion chromatography, were described in $[48,49]$. As a result of computer modeling, the IONCHROM C software (Fig. 8) was developed [50].

Using the IONCHROM software, problems of multicolumn IC with isocratic and gradient elution were considered [51, 52]. For the determination of the parameters of a new chromatographic system, necessary for modeling, its dynamic map is built, by which the conditions of the required separation in different $\mathrm{pH}$ and concentration ranges are calculated, after which three or four experiments with the chosen eluents are sufficient to correct the parameters. The model refined with the found parameters is used to predict the behavior of the system and, in particular, to optimize the analysis [53].

\section{OPTIMIZATION AND DYNAMIC MAP OF A CHROMATOGRAPHIC SYSTEM}

An approach to the description of a multicomponent MP was used to optimize HPLC analysis by an economics criterion [54]. Based on example of a chromatographic experiment from a published source, reference parameters of an HPLC system used in there were found and the optimum composition of the MP 


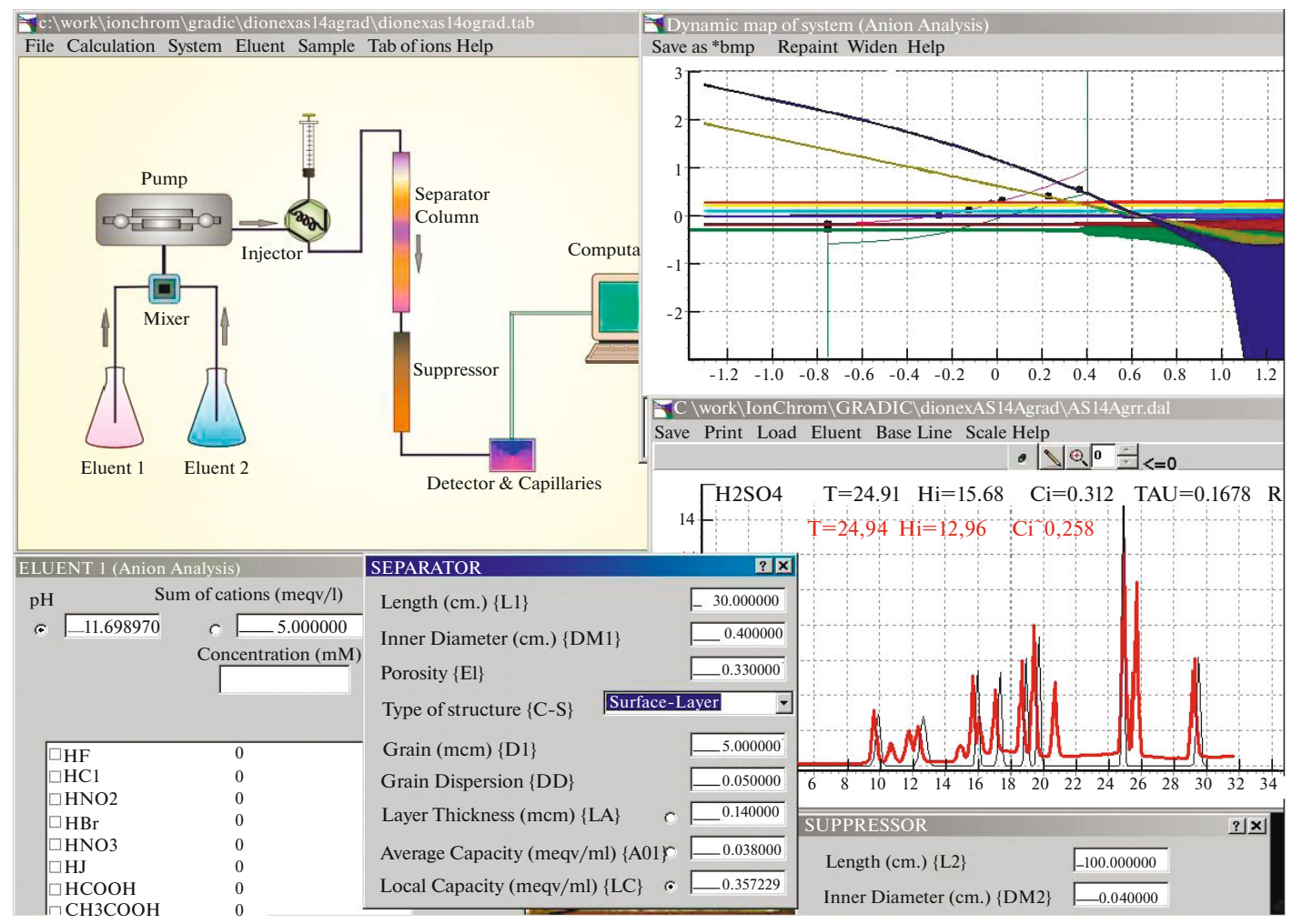

Fig. 8. Interactive interface of the IONCHROM software with open windows of eluent separation columns, suppressor, dynamic map of the chromatographic system, and the fields of the theoretical and experimental chromatograms.

for carrying out the described experiment, with which the cost of analysis was reduced twofold, was calculated.

The choice of chromatographic phases is not limited by the attainment of the required selectivity. Also research into the kinetics of sorption are performed for optimizing stationary phases by their resolving capacity and the rate of separation. To compare a new SP, developed for analytical chromatography, a new criterion, characterizing the efficiency and rapidity of the $\mathrm{SP}$, was proposed [55]:

$$
\rho=\frac{N}{D_{m} t_{\mathrm{an}}} d^{2}
$$

where $D_{m}$ is the diffusion constant of a component in the MP, $t_{\text {an }}$ and $N$ are the duration and efficiency of the determination of the component, and $d$ is the mesh of column packing. The meaning of the characteristic of the SP by efficiency and performance is given the name "sorbent performance coefficient:" the higher the value of the criterion, the higher the product of efficiency on the throughput of analysis by the time of longitudinal diffusion along the sorbent grain size.

The choice of the best conditions for the separation of mixtures taking into account the selectivity and efficiency of the chromatographic system is the central problem of optimization in chromatography. In the published data, a set of optimality criterions aimed at the achievement of the best separation within a certain time of analysis, the maximum capacity of analysis, etc., was developed $[3,56]$. An important criterion of optimization is the concept of peak capacity, introduced in the Kaiser works [57], as the number (it may be non-integer) of additional peaks that can be placed in chromatogram ranges free from peaks. The search for a minimum of peak capacity corresponds to the aim of increasing the performance of chromatography. However, peak capacity is not a very convenient optimization criterion, because precisely zero peak capacity is almost unattainable, and the result with nonzero peak capacity is degenerate and requires additional criteria. A more precise criterion is the duration of a single analysis, which is minimized in the fulfillment of requirements for the degree of component separation $[48,51]$. The effective use of this criterion requires the availability of a mathematical model of the chromatographic method, leading to the creation of a dynamic map of a chromatographic system.

The system of equations of a model of liquid chromatography can be presented in a graphical form, as a dynamic map of a chromatographic system (DMCS), which is convenient as a characteristic of the chromatographic behavior of the components of interest and an instrument for the search for the optimum separation modes. 
The abscissa in the dynamic map is $X=\log \varphi$,here the eluotropic force of MP $\varphi$ is calculated by Eq. (7). The logarithm of relative retention is plotted on the axis of ordinates (axis $y$ ) if the characteristic of the benchmark component is taken equal to zero:

$$
y_{i}=\log \left(k_{i} / k_{R}\right) \text {. }
$$

For each sample component, in the DMCS there is a corridor, constructed around the medium line $y_{i}(X)$, described by the equation:

$$
y_{i}=n_{i} \log K_{i}-\left(n_{i}-1\right) X,
$$

where unit values of the constant and charge are considered for the BC.

The borders of the corridor are related to the characteristics of the chromatogram, i.e., corrected retention time $t_{i}^{\prime}$ and peak half-width $\tau_{i}=\sigma_{i} \sqrt{8 \ln 2} \approx 2.35 \sigma_{i}$, by the equation

$$
\begin{gathered}
y_{i \pm}=y_{i}+\log \left(1 \pm \delta_{i}\right), \\
\delta_{i}=R_{i} \tau_{i} / t_{i}^{\prime} \approx R_{i} \sqrt{8 \ln 2 / N_{i}} \ll 1,
\end{gathered}
$$

where $\delta_{i} \approx 2.35 R_{i} / \sqrt{N_{i}}$ is the half-width of $i$-th corridor, $R_{i}$ is the degree of peak resolution (the required degree of separation of a component with the

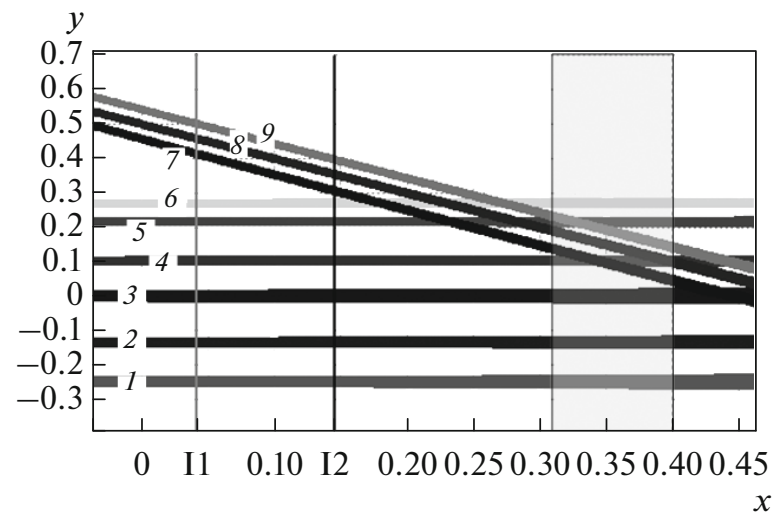

Fig. 9. Dynamic map of a chromatographic system for the separation anion mixtures by the criterion $R=1$ : (1) $\mathrm{F}^{-}$,

(2) $\mathrm{HCOO}^{-}$, (3) $\mathrm{Cl}^{-}$, (4) $\mathrm{NO}_{2}^{-}$, (5) $\mathrm{Br}^{-}$, (6) $\mathrm{NO}_{3}^{-}$, (7) $\mathrm{PO}_{4}^{3-}$, (8) $\mathrm{PO}_{3} \mathrm{~F}^{2-}$, (9) $\mathrm{SO}_{4}^{2-}$ at $\mathrm{pH} 10.82$ and the following characteristics: separation column $180 \times 3 \mathrm{~mm}$; sorbent with the structure of the surface-grafted AS-14a ionite, mesh $5 \pm 0.5 \mu \mathrm{m}$, thickness of spherical shell $140 \mathrm{~nm}$, capacity $0.038 \mathrm{mEq} / \mathrm{mL}$; ASRS-ULTRA2-mm suppressor electrodialyzer; flow rate of carbonate eluent $0.5 \mathrm{~mL} / \mathrm{min}$. [59]. Vertical lines correspond to two isocratic modes: I1, to the experiment presented in the DIONEX catalog; I2, to the optimum eluent under the specified conditions. The shaded rectangle (in the region forbidden for the isocratic mode with the criterion $R=1$ ) corresponds to the borders of the weak gradient "lens" mode shown in Fig. 10. neighbors). The zones of corridor crossing in the DMCS correspond to peaks in the chromatogram that are not separated to a necessary degree.

As follows from Eq. (10), analytes with different charges $n_{i}$ can in all cases be separated by selecting a corresponding mode in the dynamic map, because their corridors are at different angles and, starting from certain point, do not intersect. We can also note that, on different sides from the point of intersection of medium lines, there are areas of conditions with different elution orders of the components. The medium lines of two equally charged ions of a sample are parallel, so that their elution order cannot be changed, and only band width depends on the eluent strength.

A DMCS for a mixture of anions in a model system with characteristics of a DIONEX (United States) ion chromatograph of is shown in Fig. 9 [58].

Gradient elution is the convenient method of analysis of mixtures with components strongly differing on retention. Simple models of retention, ensuring calculations of unicomponent eluents and linear gradients, were developed in not numerous works on modeling of gradient chromatography $[4,5]$. However, the level of these models is obviously insufficient for the consideration of complex gradient modes; the degree of the separation of sample components attained in the calculations is taken into account poorly, which strongly depreciates the role of modeling in the development of procedures and in analysis (correspondingly, in solving of direct and inverse problems). The main equa-

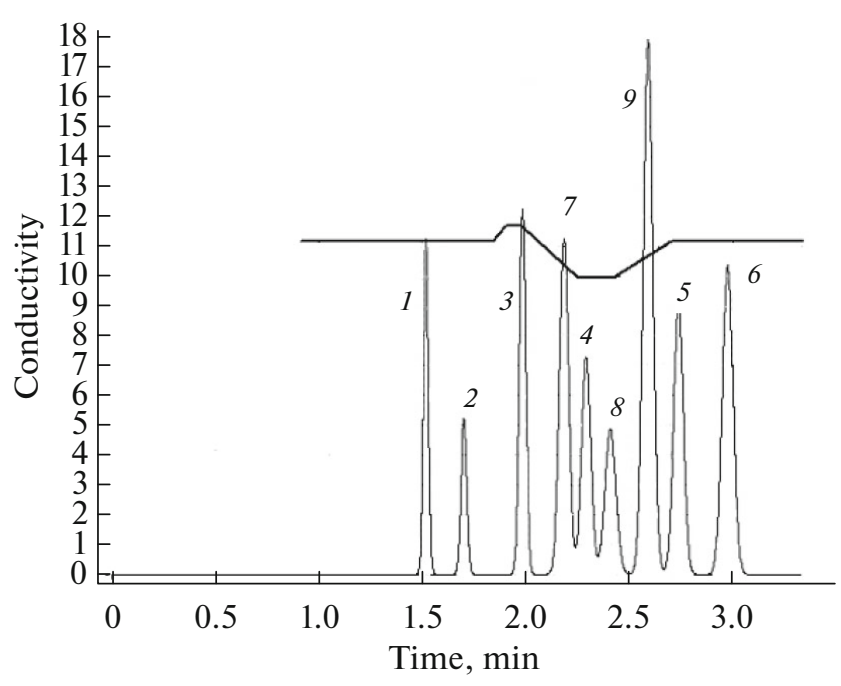

Fig. 10. A theoretical chromatogram calculated for the "lens" mode of the separation of mixture of nine anions

[(1) $\mathrm{F}^{-}$, (2) $\mathrm{HCOO}^{-}$, (3) $\mathrm{Cl}^{-}$, (4) $\mathrm{NO}_{2}^{-}$, (5) $\mathrm{Br}^{-}$, (6) $\mathrm{NO}_{3}^{-}$, (7) $\mathrm{PO}_{4}^{3-},(8) \mathrm{PO}_{3} \mathrm{~F}^{2-}$, (9) $\mathrm{SO}_{4}^{2-}$ ] on an ICS-5000 system with column AS-14a. The system in the isocratic mode I2 (see caption to Fig. 9) can separate the mixture within as short as $9 \mathrm{~min}$. with the elution order of the components presented above. 
tions for the equilibrium and kinetic characteristics of gradient IC were given in [7, 53]; examples of solutions of direct and inverse problems for systems of DIONEX ion chromatographs using both isocratic and gradient elution were presented. Further research [59, 60] showed that the accuracy of the description of gradient chromatography can be improved. The level of refinement of the model of gradient chromatography in the IONCHROM software is rather high for the consideration of alternating gradient modes [61, 62]. Figure 10 presents an example of a chromatogram calculated for the alternating "lens" mode. leading to the complete separation of a mixture of anions within threefold shorter time than in the best of isocratic mode in the same system.

Work [62] indicated a possibility of the creation of a universal algorithm for solving an extremely complex problem of the optimization of the gradient mode of ion chromatography and HPLC on an example of the so-called "weak" gradient mode.

All important components and logical relations of the developed Mathematical Chromatograph software were characterized in the presentation [63], and the main references, some developments and software are given on the project website https://www.grompiac.com.

$$
* * *
$$

The Mathematical Chromatograph project includes mathematical models of analytical methods of gas, liquid, and ion chromatography. A theoretical basis for the development of high-level models, requiring no adjustable parameters and allowing mathematical experiments in wide limits, is created. The authors believe that a combination of the presented abundant arsenal of developments in the field of modeling of methods of analytical chromatography in a uniform program has no fundamental hindrances and will require rather short time.

\section{OPEN ACCESS}

This article is licensed under a Creative Commons Attribution 4.0 International License, which permits use, sharing, adaptation, distribution and reproduction in any medium or format, as long as you give appropriate credit to the original author(s) and the source, provide a link to the Creative Commons licence, and indicate if changes were made. The images or other third party material in this article are included in the article's Creative Commons licence, unless indicated otherwise in a credit line to the material. If material is not included in the article's Creative Commons licence and your intended use is not permitted by statutory regulation or exceeds the permitted use, you will need to obtain permission directly from the copyright holder. To view a copy of this licence, visit ttp://creativecommons. org/licenses/by/4.0/.

\section{REFERENCES}

1. "Clarity" software product for processing chromatographic data. https://downloads.dataapex.com/documentation/clarity/manuals/solutions/clarity-demo.pdf. Accessed April 1, 2021.

2. "Chromeleon" software product for processing chromatographic data. https://assets.thermofisher.com/ TFS-Assets/CMD/Product-Guides/QS-7229-0004Chromeleon-7-2-QS72290004-EN.pdf. Accessed April 1, 2021.

3. Schoenmakers, P.G., Optimization of Chromatographic Selectivity, Amsterdam: Elsevier, 1986.

4. Snyder, L.R. and Dolan, J.W., High-Performance Gradient Elution: The Practical Application of the LinearSolvent-Strength Model, Hoboken: Wiley, 2007.

5. Madden, J.E., Avdalovic, N., Haddad, P.R., and Havel, J., J. Chromatogr. A, 2001, vol. 910, p. 173.

6. Dolgonosov, A.M., Nespetsificheskaya selektivnost'v probleme modelirovaniya vysokoeffektivnoi khromatografii (Nonspecific Selectivity in the Problem of Simulation of High Performance Chromatography), Moscow: KRASAND, 2013, 2nd ed.

7. Dolgonosov, A.M., Rudakov, O.B., and Prudkovskii, A.G., Kolonochnaya analiticheskaya khromatografiya: praktika, teoriya, modelirovanie (Column Analytical Chromatography: Practice, Theory, Modeling), St. Petersburg: Lan', 2015.

8. Glueckauf, E., Trans. Faraday Soc., 1955, vol. 51, p. 34.

9. Van Deemter, J.J., Znideveg, F.J., and Klinkenberg, A., Chem. Eng. Sci., 1956, vol. 5, p. 271.

10. Dolgonosov, A.M., Zh. Fiz. Khim., 1994, vol. 68, no. 12, p. 2187.

11. Dolgonosov, A.M., J. Phys. Chem. B, 1998, vol. 102, no. 24, p. 4715.

12. Dolgonosov, A.M., Russ. J. Phys. Chem. A, 2000, vol. 74, no. 2 (suppl.), p. 324.

13. Dolgonosov, A.M., Russ. J. Phys. Chem. A, 2008, vol. 82, no. 12, p. 2079.

14. Dolgonosov, A.M., Model' elektronnogo gaza i teoriya obobshchennykh zaryadov dlya opisaniya mezhatomnykh sil $i$ adsorbtsii (Electron Gas Model and Generalized Charge Theory for Describing Interatomic Forces and Adsorption), Moscow: LIBROKOM, 2009.

15. Pertsin, A.I. and Kitaigorodsky, A.I., The Atom-Atom Potential Method in the Physics and Chemistry of Organic Molecular Solids, Berlin: Springer, 1986.

16. Avgul', N.N., Kiselev, A.V., and Poshkus, D.P., $A d-$ sorbtsiya gazov i parov na odnorodnykh poverkhnostyakh (Adsorption of Gases and Vapors on Homogeneous Surfaces), Moscow: Khimiya, 1975.

17. Buryak, A.K., Russ. Chem. Rev., 2002, vol. 71, p. 695.

18. Dolgonosov, A.M., Russ. J. Phys. Chem. A, 2001, vol. 75 , no. 10 , p. 1659 .

19. Dolgonosov, A.M., Russ. J. Phys. Chem. A, 2002, vol. 76, no. 6, p. 993.

20. Dolgonosov, A.M., Russ. J. Phys. Chem. A, 2002, vol. 76, no. 12, p. 2015.

21. Dolgonosov, A.M., Theor. Chem. Acc., 2020, vol. 139, p. 90.

22. Dolgonosov, A.M., Struct. Chem., 2021, vol. 32, p. 329. 
23. Dolgonosov, A.M., Russ. Chem. Bull., 2016, vol. 65, no. 4, p. 952.

24. Dolgonosov, A.M., Russ. J. Phys. Chem. A, 1998, vol. 72 , no. 1 , p. 91 .

25. Dolgonosov, A.M., Prudkovskii, A.G., and Rudenko, B.A., in 100 let khromatografii (100 Years of Chromatography), Rudenko, B.A., Ed., Moscow: Nauka, 2003.

26. Prudkovskii, A.G., J. Anal. Chem., 2005, vol. 60, no. 7, p. 644 .

27. Pauling, L., Nature, 1948, vol. 161, p. 707.

28. Sokolov, N.D., Usp. Fiz. Nauk, 1955, vol. 57, no. 2, p. 205.

29. Hobza, P., Ann. Rep. Prog. Chem. Sect, 2004, vol. 100, p. 3.

30. Abraham, M.H., J. Phys. Org. Chem., 1993, vol. 6, p. 660 .

31. Oliveira, B.G. and Vasconcellos, M.L.A.A., J. Mol. Struct: THEOCHEM, 2006, vol. 774, nos. 1-3, p. 83.

32. Dannenberg, J.J., Haskamp, L., and Masunov, A., J. Phys. Chem. A, 1999, vol. 103, p. 7083.

33. Dolgonosov, A.M., Russ. J. Inorg. Chem., 2015, vol. 60, no. 2, p. 194.

34. Dolgonosov, A.M., Russ. J. Inorg. Chem., 2017, vol. 62, no. 3 , p. 344 .

35. Dolgonosov, A.M., Russ. J. Inorg. Chem., 2019, vol. 64, no. 4 , p. 488 .

36. Dolgonosov, A.M., J. Struct. Chem., 2019, vol. 60, no. 11, p. 1693.

37. Dolgonosov, A.M., J. Struct. Chem., 2020, no. 7, p. 1045 .

38. Dolgonosov, A.M., Sorbtsionnye Khromatogr. Protsessy, 2020, vol. 20, no. 3 , p. 343 .

39. Abraham, M.H., Ibrahim, A., and Zissimos, A.M., J. Chromatogr. A, 2004, vol. 1037, p. 29.

40. Sevcik, J. and Lowentap, M.S.H., J. Chromatogr. A, 1981, vol. 217, p. 139.

41. Zaitseva, E.A. and Dolgonosov, A.M., Sorbtsionnye Khromatogr. Protsessy, 2019, vol. 19, no. 5, p. 525.

42. Dolgonosov, A.M. and Zaitceva, E.A., J. Struct. Chem., 2020, vol. 61, no. 8, p. 1233.

43. Dolgonosov, A.M. and Zaitceva, E.A., J. Anal. Chem., 2020, vol. 75 , no. 12 , p. 1599.

44. Dolgonosov, A.M., Sorbtsionnye Khromatogr. Protsessy, 2011, vol. 11, no. 4, p. 449.

45. LePree, J.M. and Cancino, M.E., J. Chromatogr. A, 1998, vol. 829, p. 41.
46. Ponomarev, F.V. and Dolgonosov, A.M., Sorbtsionnye Khromatogr. Protsessy, 2018, vol. 18, no. 5, p. 646.

47. Dolgonosov, A.M., Sorbtsionnye Khromatogr. Protsessy, 2013, vol. 13, no. 2, p. 141.

48. Dolgonosov, A.M., Senyavin, M.M., and Voloshchik, I.N., Ionnyi obmen i ionnaya khromatografiya (Ion Exchange and Ion Chromatography), Moscow: Nauka, 1993.

49. Prudkovskii, A.G. and Dolgonosov, A.M., J. Anal. Chem., 1999, vol. 54, no. 2, p. 104.

50. Prudkovskii, A.G. and Dolgonosov, A.M., RF Patent 2000610520, 2000.

51. Dolgonosov, A.M. and Prudkovskii, A.G., J. Anal. Chem., 2002, vol. 57, no. 12, p. 1089.

52. Dolgonosov, A.M., Prudkovskii, A.G., and Kolotilina, N.K., J. Anal. Chem., 2007, vol. 62, no. 11, p. 1046.

53. Dolgonosov, A.M., Prudkovskii, A.G., and Kolotilina, N.K., J. Anal. Chem., 2016, vol. 71, no. 7, p. 703 .

54. Ponomarev, F.V. and Dolgonosov, A.M., Sorbtsionnye Khromatogr. Protsessy, 2018, vol. 18, no. 1, p. 15.

55. Dolgonosov, A.M., J. Anal. Chem., 2019, vol. 74, no. 4, p. 376 .

56. Rudenko, B.A. and Rudenko, G.I., Vysokoeffektivnye khromatograficheskie protsessy (High-Performance Chromatographic Processes), 2 vols., Moscow: Nauka, 2003, vol. 1.

57. Kaiser, R., Z. Anal. Chem., 1962, vol. 189, p. 1.

58. Thermo Scientific (DIONEX). Specification Sheet: IonPac AS14A Anion-Exchange Column. http://www. thermofisher.com/order/catalog/product/056901\#/ 056901. Accessed April 1, 2021.

59. Prudkovskii, A.G., Dokl. Math., 2013, vol. 88, p. 700.

60. Prudkovskii, A.G., Vychisl. Metody Program., 2013, vol. 14.

61. Dolgonosov, A.M. and Prudkovskii, A.G., Dokl. Chem., 2013, vol. 449, p. 49.

62. Dolgonosov, A.M. and Prudkovskii, A.G., Sorbtsionnye Khromatogr. Protsessy, 2020, vol. 20, no. 5, p. 572.

63. Dolgonosov, A.M., Abstracts of Papers, IV Vseross. konf. "Analiticheskaya khromatografiya $i$ kapillyarnyi elektroforez" (IV All-Russian Conf. on Analytical Chromatography and Capillary Electrophoresis), Krasnodar, 2020, p. 107. http://www.grompiac.com/presentations. Accessed April 1, 2021.

Translated by E. Rykova 